\title{
A mechanical device for the rapid removal and freezing of liver or brain tissue from unanaesthetized and nonparalyzed rats.
}

Quistorff, Bjørn

Published in:

Analytical Biochemistry

Publication date:

1975

Document version

Publisher's PDF, also known as Version of record

Citation for published version (APA):

Quistorff, B. (1975). A mechanical device for the rapid removal and freezing of liver or brain tissue from unanaesthetized and nonparalyzed rats. Analytical Biochemistry, 68(1), 102-219. 


\title{
A Mechanical Device for the Rapid Removal and Freezing of Liver or Brain Tissue from Unanaesthetized and Nonparalyzed Rats
}

\author{
BJ $\phi$ RN QUISTORFF \\ Department of Biochemistry, Panum Instituttet, Copenhagen, Denmark
}

Received January 22, 1975; accepted March 31, 1975.

\begin{abstract}
A mechanical, pneumatically operated device has been developed that permits withdrawal and freezeclamping of either liver or brain tissue from unanaesthetized nonimmobilized rats. Furthermore, regional sampling from brain is possible. The apparatus works in the following way: By means of rotating knives, two cross-sections are made through a rat in such a way that the slice cut out of the animal between the two knives will contain the desired tissue. This slice is then freezeclamped between precooled aluminium blocks. The sampling procedure works automatically; i.e., when the rat is in position in the apparatus and the precooled aluminium blocks have been mounted on the arms of a pair of cooling tongs, the biopsy is made simply by pressing a button. The time for making a biopsy amounts to $0.10 \mathrm{sec}$. The brain or liver tissue can be isolated from the freezeclamped slices at the temperature of liquid $\mathrm{N}_{2}$, using a small buzz saw developed for this purpose.
\end{abstract}

There is a need in biochemistry for sampling and fixation of tissue by techniques that are rapid enough to prevent the effect of ischaemia (1-3) and that permits sampling from unanaesthetized animals in order to exclude the effect of anaesthesia on metabolism (4,5).

The freezeclamping technique described by Wollenberger et al. (6) complies with the requirements as regards rapid fixation of metabolism in a tissue sample. The problem, however, is to perform tissue sampling from unanaesthetized animals rapidly enough to prevent occurrence of artefacts during the unavoidable ischaemia period, i.e., the period from interruption of circulation to the tissue sample until metabolism is stopped by freezeclamping.

A very simple approach to this problem has been made recently by Faupel et al. (3) on rat liver. For rat brain tissue, a very rapid technique has just been developed by Veech et al. (7)

The present paper describes an apparatus whereby sampling and freezeclamping of either liver or brain tissue from unanaesthetized, nonimmobilized rats is possible in $0.1 \mathrm{sec}$. Furthermore, the apparatus permits regional sampling from the brain.

A technique to disect the freezeclamped biopsies, at the temperature 
of liquid $\mathrm{N}_{2}$, using a small buzz saw, is described. A preliminary report on the sampling apparatus has appeared (8) and measurements on brain samples have been described (9-13).

\section{Materials}

The knife blades for the sampling apparatus and the saw blade for the buzz saw were specially fabricated by the firm G. Nelholdt, Copenhagen, Denmark. All other equipment for the apparatus was specially made in the workshop of the Institute.

\section{Animals}

Male and female Wistar rats, weighing 180-300 g, have been used for brain or liver sampling. A weight of $200 \mathrm{~g}$ is found to be preferable with the size of tube applied (see later). No special technique is necessary to make the rats enter the tube of the apparatus. About $80 \%$ of them will enter voluntarily just when held in front of the tube. However, most of these rats behave in a stressed manner (excretion of faeces and urine). The stress reaction can be prevented if the animals are subjected to the following "training program" before the actual experiment: For at least a week they are kept in the laboratory in two cages connected with a tube corresponding to that of the sampling apparatus. As one cage contains solid food and the other drinking water, the rats are compelled to run through the tube. In fact, after a day or two, this tube becomes the favorite residence for the rats. Besides this passive training procedure, the rats are handled once a day, during the 7-day period, exactly in the same way as on the day of the experiment; i.e., the animal is restrained in the tube of the sampling apparatus (see Fig. 8), and 10-15 min later a sham experiment is performed. Afterwards, the constriction of the tube is removed, whereby the rat is allowed to continue through the tube into the cage. Most rats behave in an unstressed way (no excretion of faeces and urine) on the second or third day they are subjected to this procedure.

It shall be emphasized that sampling and decapitation take place coinstantaneously irrespective of the kind of tissue sampled. The decapitation lasts $0.02-0.03 \mathrm{sec}$.

\section{General Construction and Function of the Sampling Apparatus}

The apparatus is shown in Fig. 1 and on a simplified diagram in Fig. 2. In both figures liver sampling is depicted, but there is no essential difference between sampling of liver and brain tissue, apart from the position of the rat in the tube (marked g,f,g in Fig. 2) and details in the construction of the aluminium blocks for the cooling tongs $(h, h$, in Fig. 2). In the following the letters $a-j$ and the numerals $1-3$ and I-III refer 


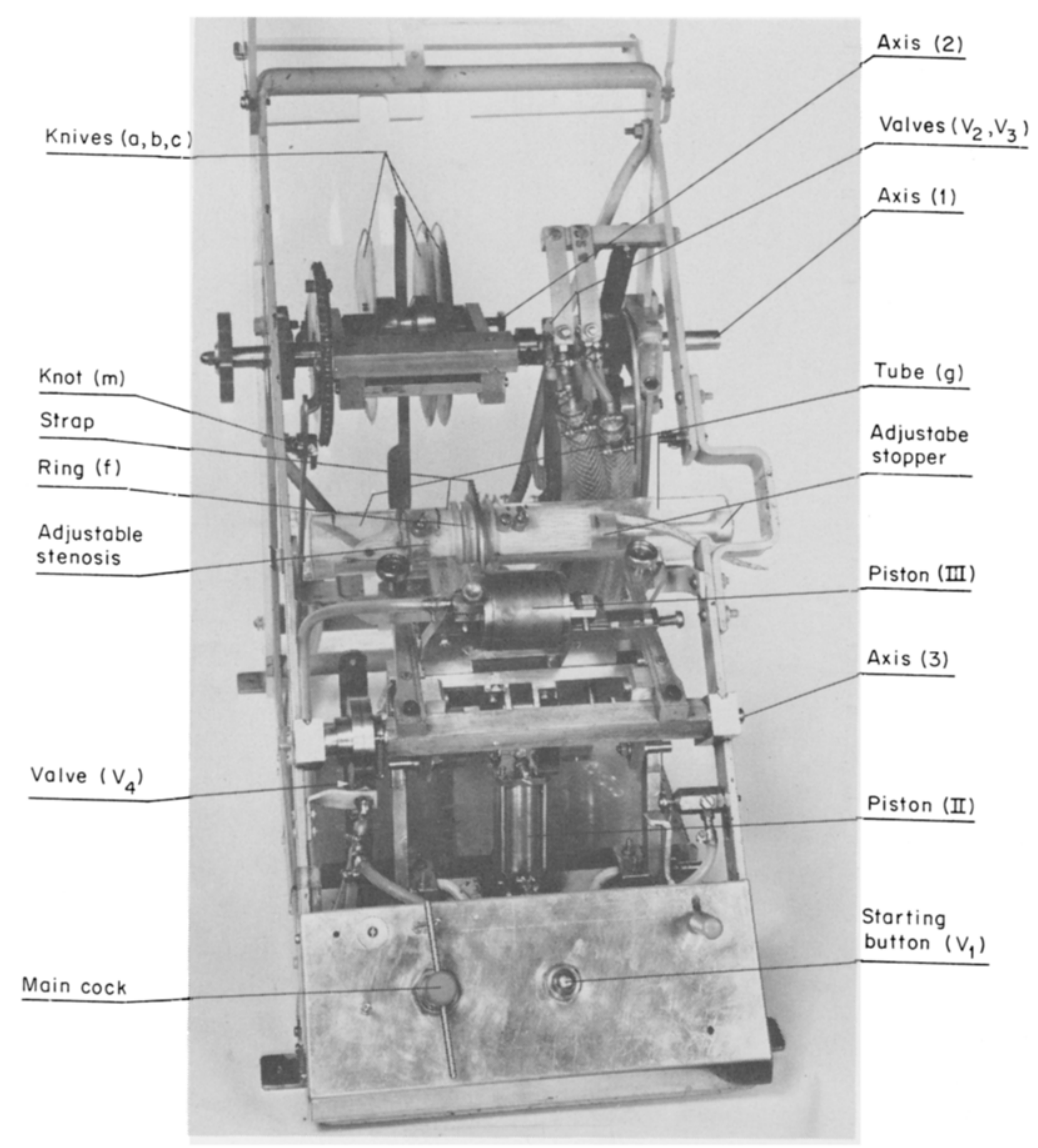

FIG. 1. The apparatus for rapid removal and freezeclamping of either rat liver or rat brain. The rat is seen in the transparent tube in position for sampling of liver tissue.

to Fig. 2 and Fig. 3 unless otherwise stated. The apparatus is operated by means of compressed air which supplies power to the three pistons, I-III. These pistons produce the movements necessary for the sampling procedure. Piston I brings about two different movements of the knives a, b and c, i.e., a cutting movement around axis 1 and a rotating movement round the axis of the knives (axis 2). Piston II moves the two precooled aluminium blocks $(h, h)$ on the cooling tongs upwards in position on each side of the "rat slice" situated in the ring (f), and simultaneously the remaining parts of the rat in the tube $(\mathrm{g}, \mathrm{g}, \mathrm{g})$ are removed. Piston III is responsible for the power for the freezeclamping of the slice. The direction of the clamping is shown by arrows on the blocks $(h, h)$ of the cooling tongs. The closing of the tongs takes place around the point $\mathrm{j}$. Only one arm moves. 


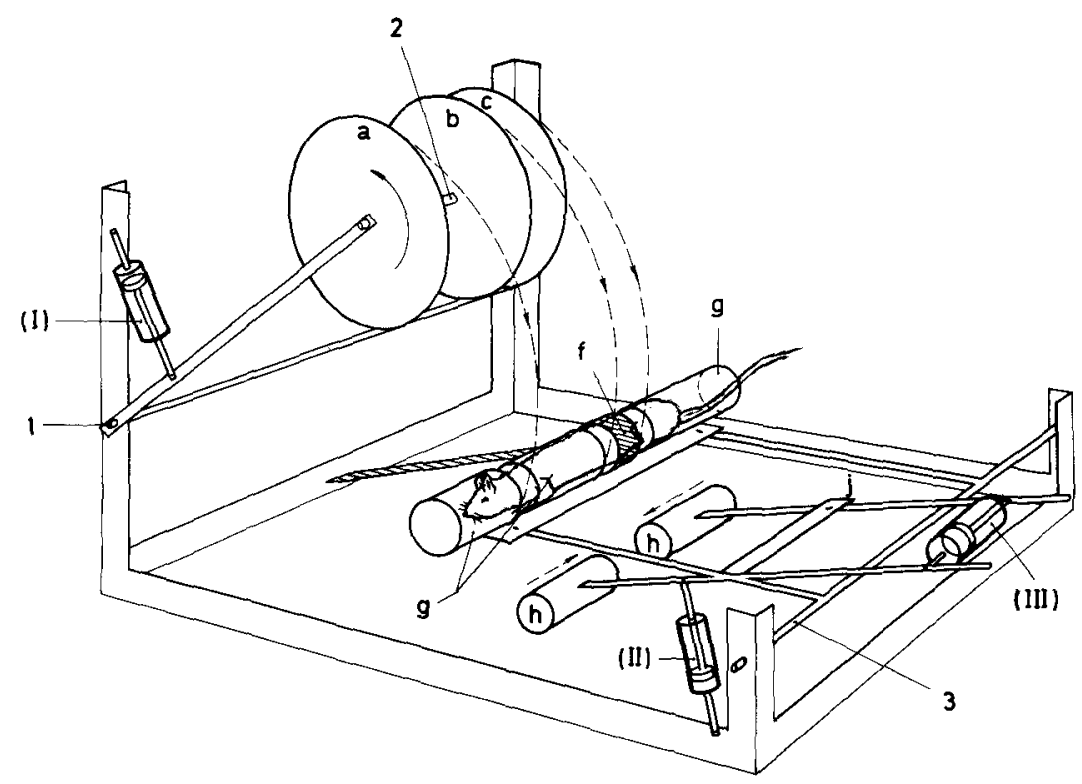

FIG. 2. Diagram of sampling apparatus. The rat is seen in the tube in position for liver sampling.

Figure 3 gives the sequence of the steps in the sampling procedure. Four consecutive situations are shown.

Situation 1, Fig. 3. The apparatus is ready for sampling. The rat is in the correct position in the tube for liver sampling (cf. Figs. 1 and 8A). This means that the neck is at the first gap in the tube corresponding to knife a, and the liver region of the animal is between the second and the third gap corresponding to the knives $b$ and $c$. For brain sampling the part of the brain to be examined is at the second gap (cf., however, The Biopsy). The cooled aluminium blocks $(\mathrm{h}, \mathrm{h})$ have been mounted on the cooling tongs. The apparatus is now activated by opening access for the compressed air to piston I (cf. Figs. 4 and $5 \mathrm{~A}$ ), whereby the three knives together will perform a circular movement of about $180^{\circ}$ around axis 1 to cleave the rat through the gaps in the tube.

Situation 2, Fig. 3. The rat is cut and knives $b$ and $c$ are still situated on each side of the liberated rat slice containing part of the liver. At this moment piston II is activated (cf. also Figs. 2 and 4A) whereby the tube $(\mathrm{g}, \mathrm{g}, \mathrm{g})$ together with the cooling tongs $(\mathrm{h}, \mathrm{h})$ is moved upwards (cf. also Fig. 8) in a circular movement around axis 3, whereas the slice remains in the short, fixed part of the tube (f) (see later), enclosed between knives $b$ and $c$.

Situation 3, Fig. 3. The cooled blocks on the arms of the cooling tongs 


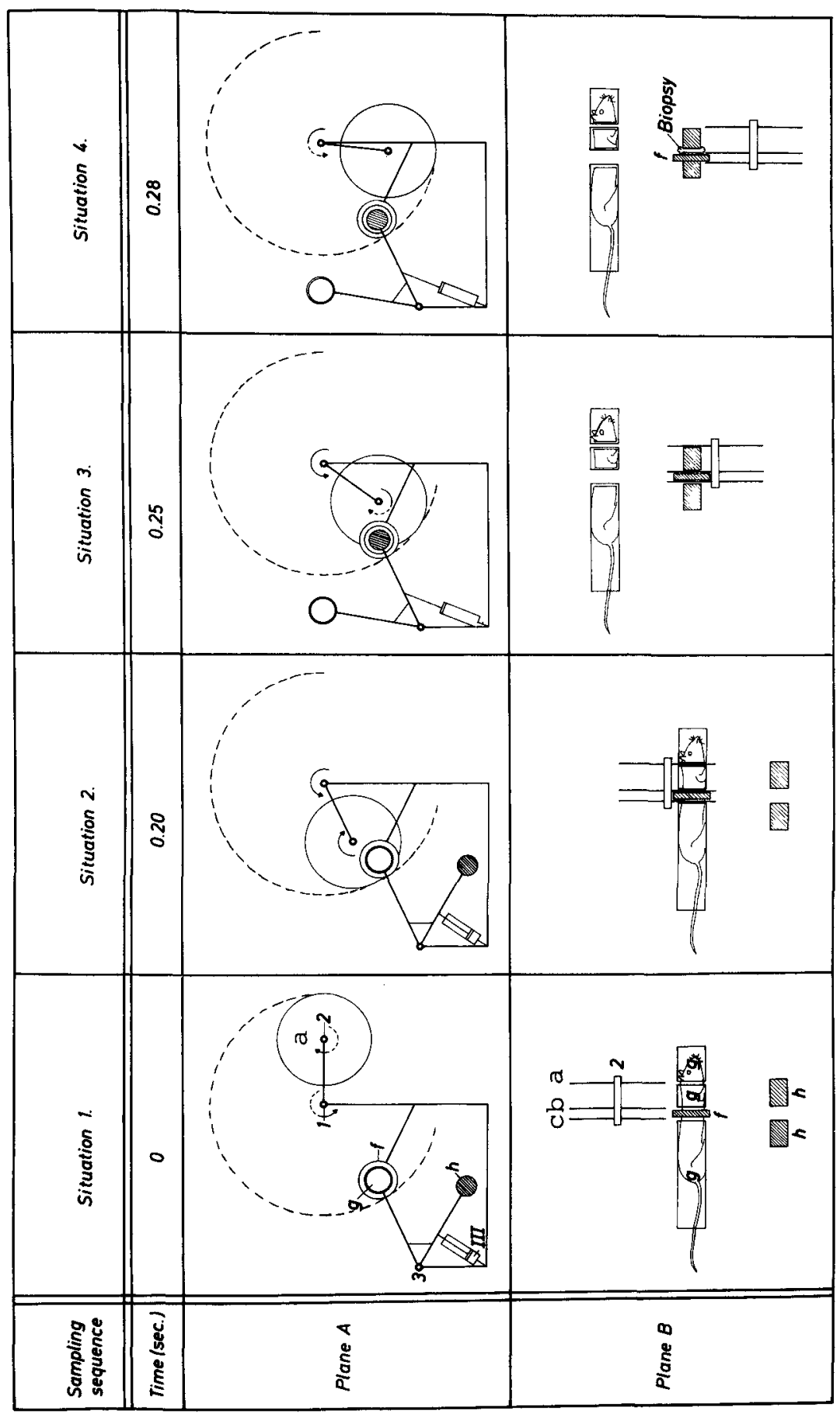


A.

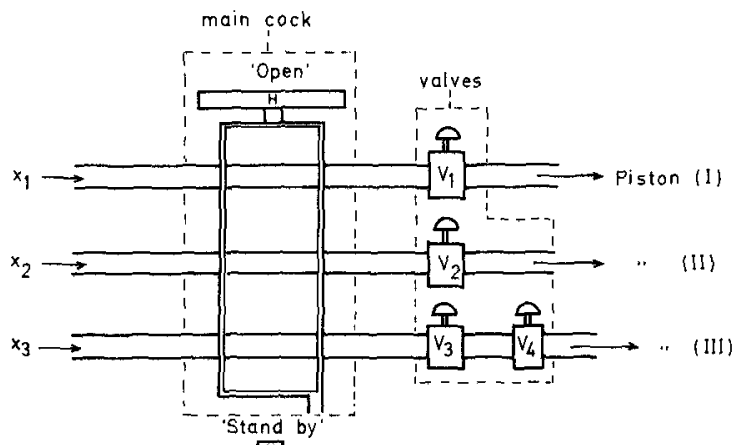

B.

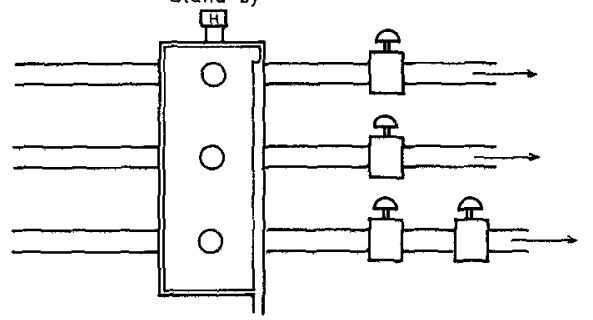

c.

\begin{tabular}{|c|c|c|c|c|}
\hline \multicolumn{2}{|c|}{ Pressure, Atm } & $x_{1}$ & $x_{2}$ & $x_{3}$ \\
\hline \multicolumn{2}{|c|}{ Liver sampling } & 6.1 & 5.5 & 3.0 \\
\hline \multirow{2}{*}{$\begin{array}{l}\text { Brain } \\
\text { sampling }\end{array}$} & 'Thin slice' & 4.0 & 5.5 & 5.0 \\
\hline & 'Thick slice' & 4.0 & 5.5 & 2.5 \\
\hline
\end{tabular}

Fig. 4. The compressed air system. (A), Diagram of the pressure circuits with the main cock in open position; (B), the main cock in stand-by position; (C) values of the compressed air pressure in the three pressure circuits.

have reached a position exactly at the level of the slice, which is still enclosed and fixed between the knives.

Situation 4, Fig. 3. At the moment the knives no longer cover the slice in the ring, piston III is activated (cf. also Fig. 4A) and the contents of the ring are freezeclamped.

\section{Description and Function of Details of the Sampling Apparatus}

The compressed air system. The compressed air system is shown in Figs. 4A and B and consists of three separate pressure circuits. In Fig. $4 \mathrm{C}$ are the values of the pressure, $x_{1}, x_{2}$ and $x_{3}$, in the three circuits used

FIG. 3. Sequence of sampling procedure. Four consecutive situations in the sampling procedure are shown on diagrams depicted in two vertical planes ( $A$ and $B$ ) perpendicular to each other. Plane $\mathbf{A}$ is perpendicular and plane $\mathbf{B}$ parallel to all three axes $(1,2,3)$. On the top of the figure the time course of the sequence is given. 
for liver and brain sampling respectively. The compressed air in each of the three circuits is supplied to the apparatus via a main cock, which has two positions: Stand-by (Fig. 4B) and open, (Fig. 4A). When in position stand-by, there is no pressure on the machinery. When open, the apparatus will function if the valve $\left(\mathrm{V}_{1}\right)$ is pressed (Figs. $4 \mathrm{~A}$ and $\mathrm{B}$; see also Fig. 1). The activation of the valves $\left(V_{2}\right.$ and $\left.V_{3}\right)$ is brought about by cams on axis 1. By adjusting these cams on the axis, each of the valves can be activated at any point in the orbit of the knives; i.e., $\mathrm{V}_{2}$ and thereby piston II is adjusted to be activated at the very moment the rat is cut through (cf. Fig. 3, Situation 2), and $V_{3}$ is adjusted to be activated when the knives are free of the clamping area (cf. Fig. 3, Situation 4). The valve $V_{4}$ is activated by the position of the blocks of the cooling tongs, namely when they are situated exactly corresponding to the ring (cf. Fig. 3, Situation 4). As a security measure, $V_{3}$ and $V_{4}$ are connected in series, thereby ensuring both that the jaws of the cooling tongs are in the correct position and that the knives are free of the clamping area before piston III can be activated.

The movement of the knives. To attain sufficient speed the knives are submitted to a hewing movement of $180^{\circ}$ before they hit the rat in the tube (cf. Fig. 3, Situations 1 and 2). How this hewing movement is brought about by means of the power from piston I is shown diagrammatically in Fig. 5A. Towards the end of the hewing movement the conveying power is gradually reduced (see Fig. 5A) and the knives therefore stop when deadpoint is passed. Figure 5B shows how the hewing movement, around axis 1 , automatically brings about a rotation of the knives around axis 2: On axies 1 and 2 are placed two sprocket wheels interlinked by a chain. The ratio of the diameter of the wheels is $1: 5$. The sprocket wheel on axis 1 is kept in a fixed position (see Fig. 5B). The hewing movement will therefore cause the knives to rotate around axis 2 in the opposite direction of the hewing movement (see Fig. 5B.) ${ }^{1}$

At the time that the rat is cut it will be an advantage if the rotation of the knives (around their own axis) is stopped, as the liberated rat slice which at that moment is situated between knives b and c (cf. Fig. 3, Situation 3) is very easily displaced. This problem is solved by letting the conveying movement, the hewing movement proper, release the sprocket wheel on axis 1 from its fixed position (cf. Fig. 5B), i.e., in

\footnotetext{
${ }^{1}$ In a former model of the machinery, presented at The Annual Meeting of The Danish Biochemical Society, Arhus, 1971, this rotation of the knives was brought about by an electromotor, and which direction of rotation was best for cutting was determined. Surprisingly, it was found almost impossible to make the knives cut the rat when rotating the "right way", i.e., the knives will throw away the object to be cut. This phenomenon is probably due to the fact that the speed of rotation of the knives will decrease because of the cutting movement of the knives. However, the result produced the idea to let conveying movement drive the knife rotation as described.
} 
A

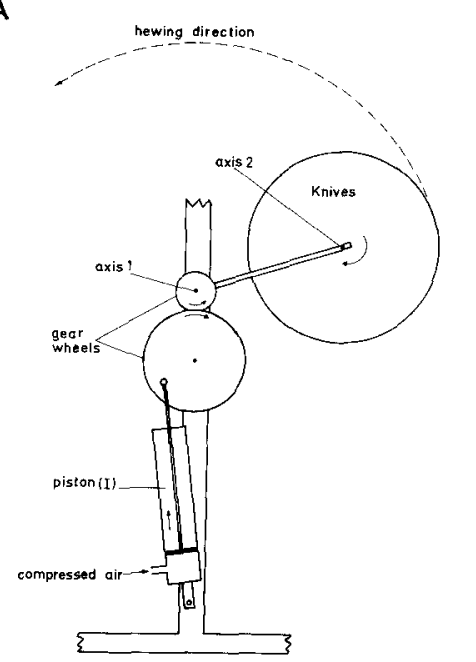

B

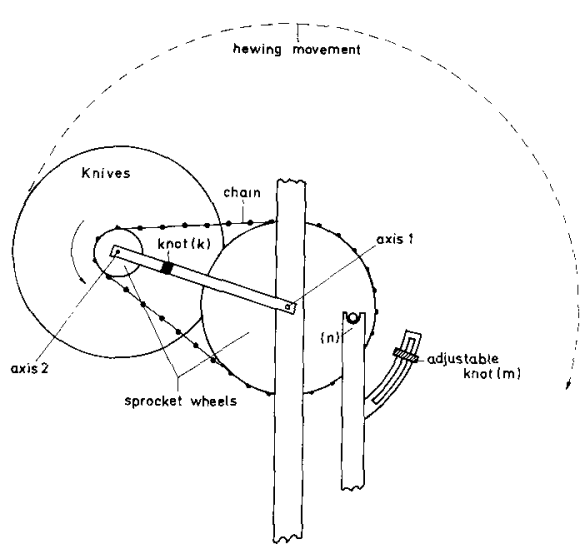

FIG. 5. The movement of the knives. (A), Diagram showing how piston I brings about the hewing movement of the knives (seen from the right side of Fig. 1); (B), how the rotation of the knives around their own axis (axis 2) is brought about by the hewing movement proper (seen from left side of Fig. 1).

passing the adjustable knot $(\mathrm{m})$, the knot $(\mathrm{k})$ will press out the strap $(\mathrm{n})$, whereby the sprocket wheel will be free to rotate. The knives are therefore no longer forced to rotate round their own axis. By adjusting knot $\mathrm{m}$, the releasing mechanism can be activated at a suitable point in the conveying orbit, making it possible to eliminate the upwards acceleration that the knives would give the slice.

The knife blades. All three knives are clamped on axis 2 (see Fig. 6 and also Fig. 2). It is possible to adjust the distance between the two knives ( $b$ and $c$ ) to any value between 4 and $14 \mathrm{~mm}$. The knives are adjusted to cut as close to the ring as possible (cf., however, Brain sampling). This is essential, especially for liver sampling, as the thin and very elastic structures (skin and fascies) inside the animal are otherwise not cut through. ${ }^{2}$ During cutting, the speed of rotation of the knives is about $600 \mathrm{rpm}$, and some $80^{\circ}$ of the knives' edges, about $13 \mathrm{~cm}$, is engaged in the cutting. This means that the knives can be used at four or five different places before they are worn down.

The biopsy slice of the rat is always cut between knives $b$ and $c$, irre-

${ }^{2}$ The cutting is considerably improved by placing a cylinder of paper around that part of the rat which is cut through, i.e., a piece of pasteboard (e.g., index card) is rolled into a cylinder (double layer) and released inside the tube of the apparatus before the rat enters. The pasteboard ring around the sample cut free from the rat will also improve the freezeclamping as it counteracts the spread of the tissue that would result from the compression. For regional brain sampling, this latter function of the paper is essential. 


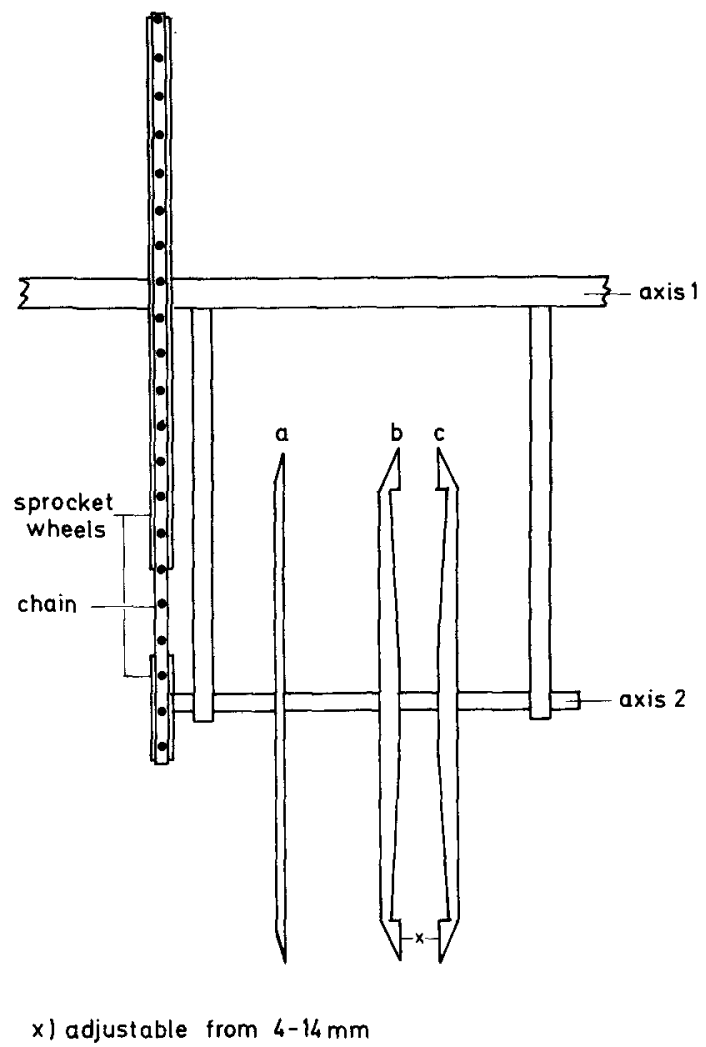

FIG. 6. The knife blades. Diagram showing the shape of the three knife blades (a,b,c) and their relation to axes 1 and 2 . The diameter of a knife blade is $175 \mathrm{~mm}$, the thickness is 1.5 $\mathrm{mm}$.

spective of the location on the rat (see Fig. 6 and also Fig. 3). Knife a is used only when liver sampling is performed to assure instant death of the animal by decapitation.

The tube and the cooling tongs. In Fig. 1 the transparent polyvinyl chloride (PVC) tube containing the rat in liver sampling position is seen. Figure 7 shows a close-up diagram of the tube and cooling tongs. The blocks of the tongs are hidden underneath the tube (cf. Fig. 3, Plane A). The tube is divided into four parts $(g, g, f, g)$ by three gaps, and, as seen in Fig. 7, the three parts and the cooling tongs are fixed in one unit which moves around axis 3 . The fourth part of the tube, the interchangeable ring (termed $f$ in Figs. 2 and 3 ) is fastened in a strap and is immovable. The strap is $3 \mathrm{~mm}$ wide and permits the fixation of rings with varied inner diameter and of different thickness, corresponding to the desired slice thickness (cf. Fig. 10). 


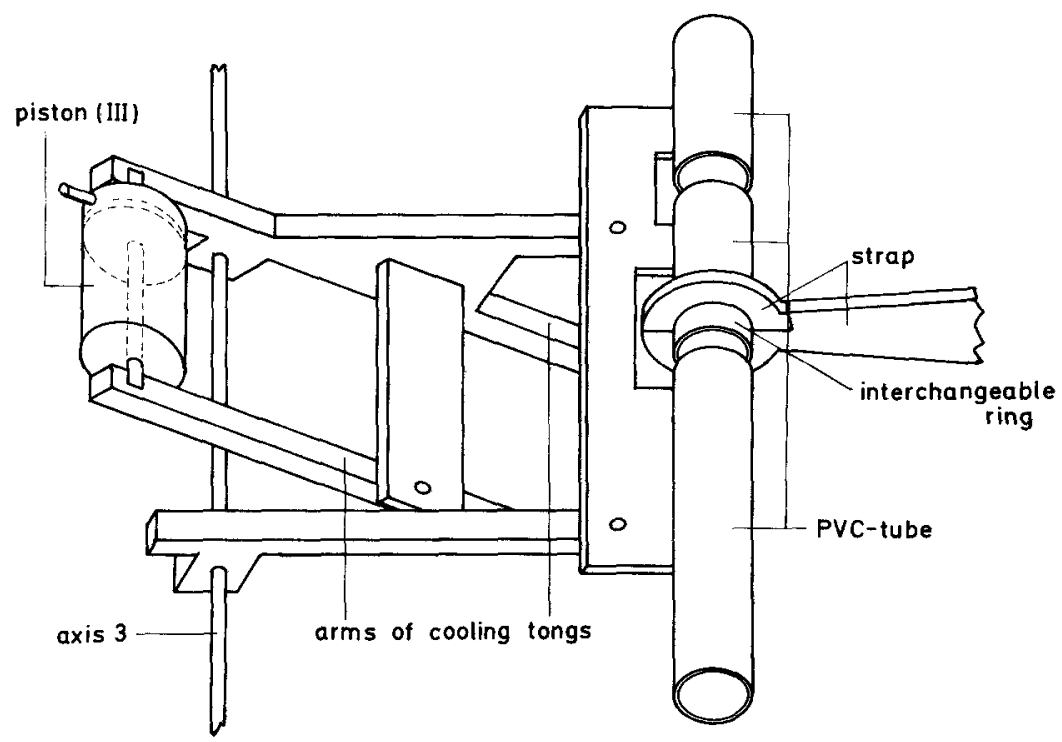

FIG. 7. The tube and the cooling tongs. Diagram of the unit consisting of cooling tongs and rat tube, in relation to the strap fit for fixation of the immovable part of the tube, i.e. the interchangeable ring (cf. Fig. 2).

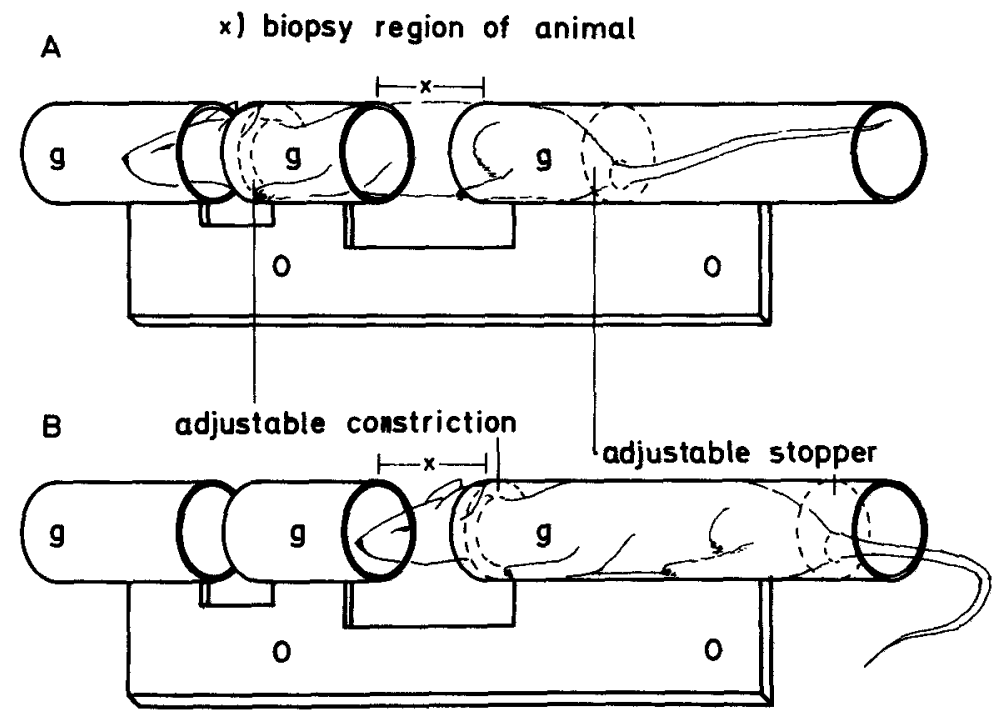

g) PVC-tube

FIG. 8. The tube. Diagram showing how the rat is kept in a fixed position in the tube. (A), In position for liver sampling; (B), In position for brain sampling. The length of the tube is $26 \mathrm{~cm}$, and the inner diameter is $4.5 \mathrm{~cm}$. 
A

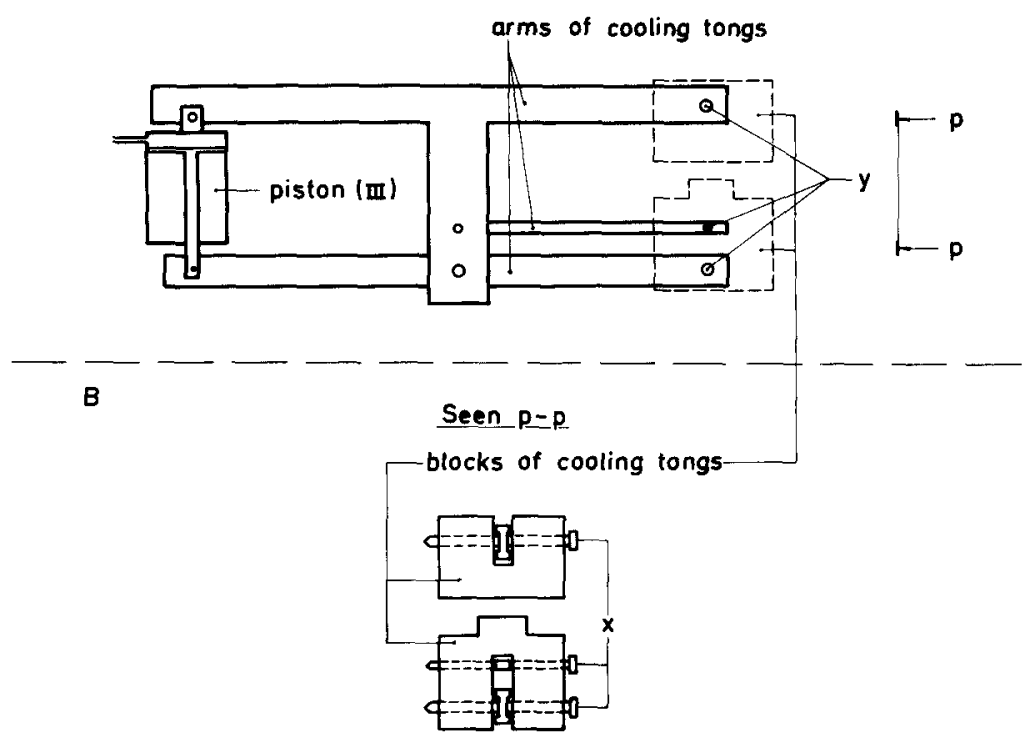

FIG. 9. The cooling tongs. (A), Diagram of the cooling tongs. (B), Diagram showing the principle of fixation of the demountable blocks on the arms of the cooling tongs (cf. Fig. 10).

For clarity, the PVC tube in Figs. $8 \mathrm{~A}$ and $\mathrm{B}$ has been depicted without the cooling tongs and the ring part of the tube. In Fig. $8 \mathrm{~A}$ an adjustable constriction is placed in such a way that the liver region of the rat is just in the right position in the tube when the shoulders encounter the constriction. The rat is fixed in this position by means of an adjustable stopper, provided with a slit for the tail (cf. Animals). In Fig. 8B the constriction and the stopper are in position for sampling of brain tissue. The gas mixture inspired by the rat in the tube can be controlled by means of a fitting system (visible in Fig. 1) after closing of the ends and the gaps of the tube with tape.

Figure 9A shows the cooling tongs. One arm is moved by piston III, while the other arm is fixed (cf. Fig. 2). The jaws of the cooling tongs, the aluminium blocks, are demountable. The advantage of demountable blocks is that they can be cooled separately in liquid $\mathrm{N}_{2}$ and mounted just before sampling is performed. About $10 \mathrm{sec}$ elapses from the time the blocks are taken from the liquid $\mathrm{N}_{2}$ and mounted on the cooling tongs until the biopsy is made. Therefore, the heat transfer from the arms of the cooling tongs to the blocks is negligible. Furthermore, the contact surfaces of between the blocks and the arms are made very small, as seen in Fig. 9B.

The freezeclamping blocks of the cooling tongs. Figures $10 \mathrm{~A}-\mathrm{C}$ show different presentations of the blocks. It appears that block $B_{1}$, to be 


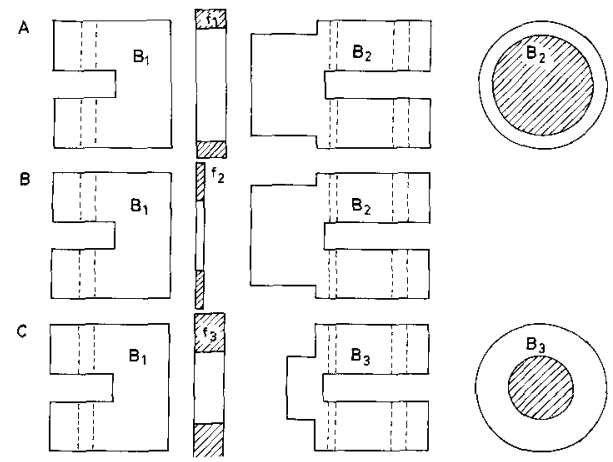

FIG. 10. The blocks of the cooling tongs. Diagram giving the shape of the blocks of the cooling tongs and of the corresponding rings. All blocks are made of aluminium, each weighing about $300 \mathrm{~g}$. (A), The blocks and the ring used for liver sampling; (B and C), blocks and rings used for brain sampling.

mounted on the immovable arm of the cooling tongs (cf. Figs. 9A and 2), is the same in all cases, whereas the shape of the other block, $B_{1}, B_{2}$ or $B_{3}$ and of the ring, $f_{1}-f_{3}$, depend on the kind of biopsy desired.

The Block $B_{2}$ and ring $f_{1}$, as shown in Fig. 10A, are used for liver sampling. The diameter of this block allows it to pass into the ring to freezeclamp the biopsy. The final thickness of the freezeclamped biopsy slice will therefore depend partly on the magnitude of pressure applied to piston III (see Fig. 4) and partly on the thickness of the slice cut out, as determined by the distance between knives b and $\mathrm{c}$ (see The Biopsy). The same blocks $\left(B_{1}\right.$ and $\left.B_{2}\right)$ can be used for brain sampling also, if in combination with a thin PVC ring, $\mathrm{f}_{2}$ (see Fig. 10B). The inner diameter corresponds to the head of the rat but is less than the diameter of movable block $B_{2}$, (see Fig. 10B). The ring will therefore be carried along and will be clamped between the two blocks when the cooling tongs close. Thus the final biopsy thickness will in this case be defined only by the thickness of the ring if sufficient clamping pressure is used.

The blocks and the ring shown in Fig. 10C are also used for brain sampling. The inner diameter of ring $f_{3}$ is equal to that of $f_{2}(28-31 \mathrm{~mm}$, depending on the size of the rat), whereas the diameter of the head of block $B_{3}$ allows it to pass into the ring (Fig. 10C). In this case the cooling tongs are adjusted to close with a suitable small force (cf. Fig. $4 \mathrm{C}$ ), and thus the slice of the rat's head will only be moderately compressed (see Brain sampling. Thick slices).

\section{The Biopsy}

Liver sampling. The distance between the two knives $\mathrm{b}$ and $\mathrm{c}$ (cf. Figs. 2, 3 and 6) cutting through the liver region is usually adjusted to fit 
a $12-\mathrm{mm}$ ring. If a rat of $210-230 \mathrm{~g}$ is used and the constriction is placed as shown in Fig. 8A, such a $12-\mathrm{mm}$ cut will pass through the central part of the liver and leave liver tissue both in the thorax and in the abdomen. Stomach and intestine can always be found undamaged in the hind part. Apart from continuous structures such as vessels, oesophagus, columna etc., the slice will only contain skin, fascie, diaphragm and liver tissue. A successful (five to ten out of ten rats) slice from a rat weighing $220 \mathrm{~g}$ will contain half of the liver. After the freezeclamping to a final biopsy thickness of 3-5 mm, usually one quarter will be left, the remaining part being pressed out during the clamping. From these 1-2 $\mathrm{g}$ of frozen liver tissue it is possible to remove between 0.2 and $1 \mathrm{~g}$, depending partly on how deep in the biopsy one wishes to sample the tissue (considerations of freezing time) and partly on how the liver tissue is scattered in each biopsy, compared with other structures (considerations of security against mixing with tissue of a different kind).

The liver tissue can be isolated from the biopsy under liquid $\mathrm{N}_{2}$ by scraping from the surface with a cooled chisel or by means of a small buzz saw (see below). In this way, several layers parallel to the freezing surfaces can be sampled from the same biopsy. Metabolic studies on such liver samples are in preparation in this laboratory.

Brain sampling. Thin slices. The thin slices, made with ring $\mathrm{f}_{2}(2.5-\mathrm{mm}$ thickness) and blocks $B_{1}$ and $B_{2}$ (Fig. 10B) can be cut out at any longitudinal level of the brain. Practice shows that it is possible in about $75 \%$ of the experiments to hit the desired location with an accuracy of \pm 1 $\mathrm{mm}$. Knives $\mathrm{b}$ and $\mathrm{c}$ are adjusted to a distance of $4.5 \mathrm{~mm}$, providing a space of $1 \mathrm{~mm}$ between the ring and each of the knives. Thus, during freezeclamping, the slice is compressed from $4.5 \mathrm{~mm}$ to the thickness of the ring, i.e., $2.5 \mathrm{~mm}$ (cf. Fig. 10B). On such a lightly compressed and frozen slice it is still possible macroscopically to recognize some of the coarse structures of the brain. Thus right and left hemispheres can be distinguished from each other, and sometimes cortex can be clearly separated from underlying structures.

Under liquid $\mathrm{N}_{2}$ the brain tissue can be separated from the remaining part of the biopsy by using small tongs cooled in liquid $\mathrm{N}_{2}$ and a small buzz saw (see below).

The advantage of using the setup for brain sampling just described (Fig. 10B) compared with that described below (Fig. 10C) is, that rapid freezing of larger amounts of tissue can be obtained because of smaller final biopsy thickness. However, it is a drawback that the knives must be extremely sharp and without any chips in the edges at all. Otherwise the architecture of the thin slice will be destroyed making it impossible to recognize the brain part of the freezeclamped slice with certainty. However, by widening the slice, as shown in Fig. 10C, this cutting 


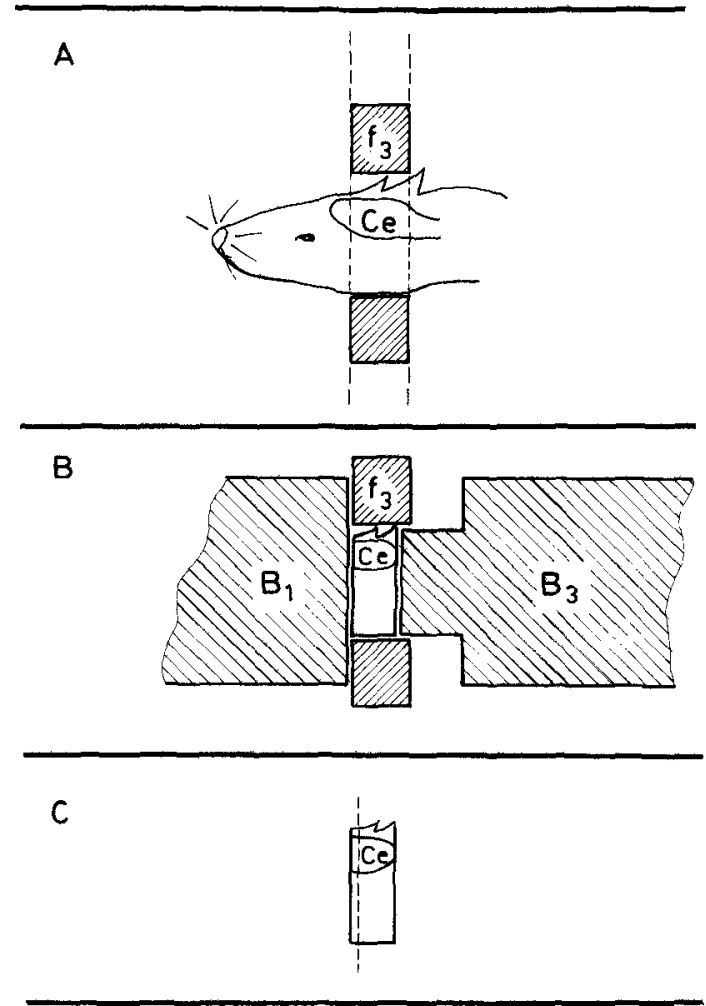

FIG. 11. Regional brain sampling. (A), The position of the head of the rat relative to the ring $\left(f_{3}\right)$. The rat is cut corresponding to the dotted lines. Ce represents the brain. (B), The head slice is freezeclamped between the precooled aluminium blocks ( $B_{1}$ and $\left.B_{3}\right) .(C), A$ thin slice is derived from the frozen tissue block by a cut corresponding to the dotted line. Different anatomical structures can be isolated from this thin slice in the frozen state.

problem becomes much less critical. For this reason, only little experience has so far been accumulated concerning thin slices.

Brain sampling. Thick slices. With the setup of Fig. $10 \mathrm{C}$ rather precise regional brain sampling can be performed. Figures $11 \mathrm{~A}-\mathrm{C}$ show a diagram of the procedure: The rat is placed in the tube so that knife $b$ corresponding to the left of the dotted lines in Fig. 11A, will pass through the part of the brain to be examined. Thereafter the released head slice, 13-mm thickness, is freezeclamped between $B_{1}$ and $B_{3}$ to a final thickness of $9-10 \mathrm{~mm}$ (Fig. $11 \mathrm{~b}$, see also footnote 2). A thin slice (or slices) can then be derived from the frozen biopsy (see below), as shown in Fig. 11C.

On the surface of the frozen biopsy which has had direct contact with block $\mathrm{B}_{1}$ (Fig. 12A), the coarse structures of the brain can be recog- 


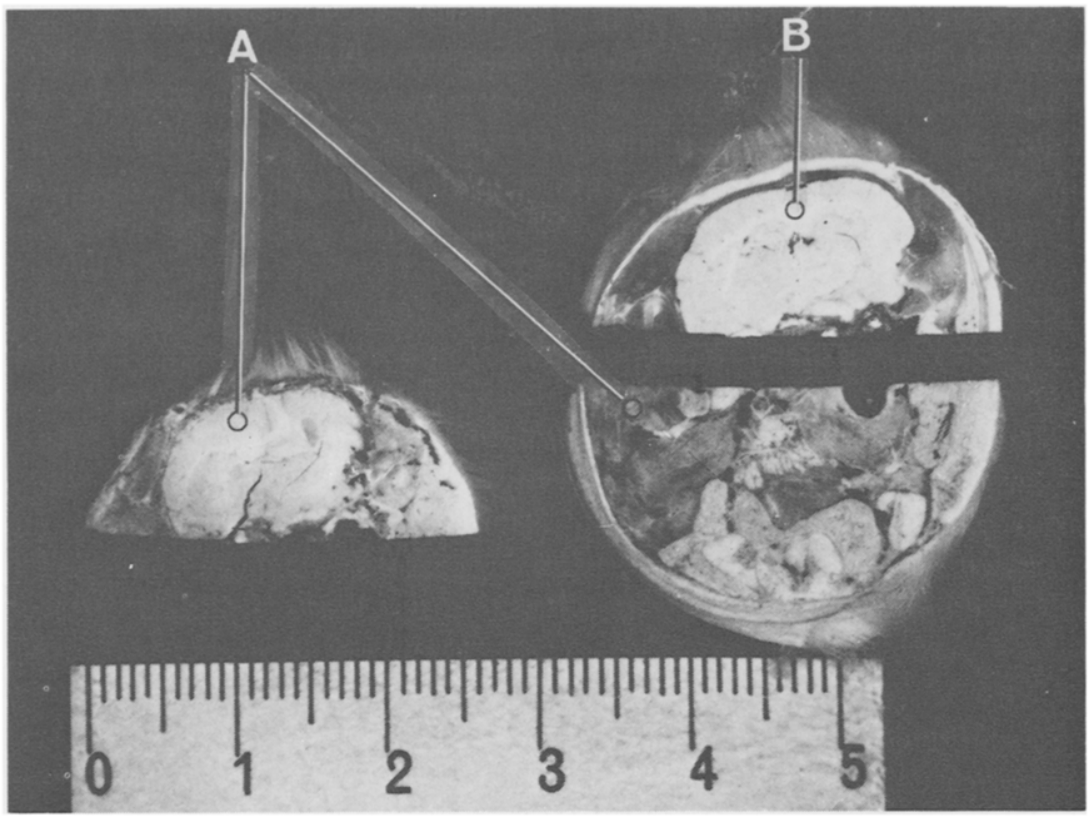

FiG. 12. The freezeclamped brain sample. (A), The surface freezeclamped against block $B_{1}$ (see Fig. 11B). (B), The sample is cut in the frozen state by means of the buzz saw (see Fig. 13). The picture shows the surface created by the saw. The scale is in centimeters.

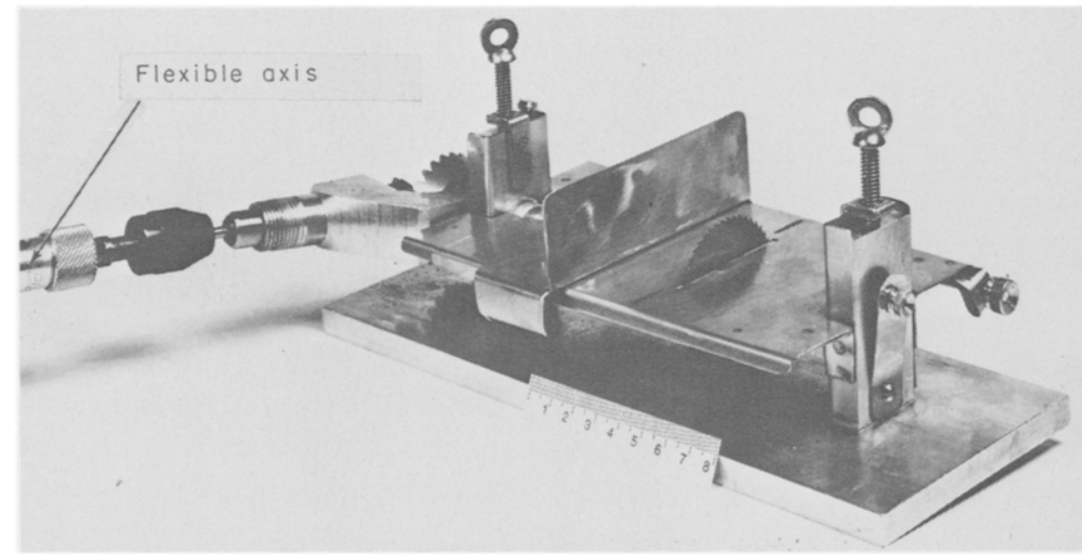

FIG. 13. The buzz saw used for dissection of the freezeclamped biopsies. The diameter of the saw blade is $50 \mathrm{~mm}$, the thickness, $0.25 \mathrm{~mm}$. The tooth of the saw blade has a size of $2.5 \mathrm{~mm}$. The scale in the figure is centimeters. 
nized, as described before (Thin slices). However, the surfaces created by the saw present all macroscopic structures even more nicely (Fig. 12B).

A major structure such as cortex can easily be picked out from the derived slice for metabolic analysis $(10,13)$, or the redox state of the different structures can be read directly from the surface of the frozen slice $(11,12)$.

\section{The Buzz Saw}

The saw shown in Fig. 13 is, in fact, an ordinary buzz saw built in small dimension and equipped with adjustable land and tabletop. The saw is operated in a refrigerated glove box at $-30-40^{\circ} \mathrm{C}$ and is driven by an electromotor from the outside via a flexible axis at a speed of $100-300 \mathrm{rpm}$. It is found that the cutting is performed best if the temperature of the tissue is close to the temperature of liquid $\mathbf{N}_{2}$. So the biopsies are stored in liquid $\mathrm{N}_{2}$ in the glove box, are taken directly from there when they are to be cut, and are furthermore cooled intermittently in liquid $\mathrm{N}_{2}$ during the process of sawing $(5-20 \mathrm{sec})$. A slice cut free from the biopsy must be of a minimum thickness of $0.9-1.1 \mathrm{~mm}$ to prevent it from falling apart during the process of sawing.

\section{Time Course of the Sampling Procedure}

All time intervals given derive from rapid motion picture films of the sampling procedure (Bolex, 64 frames/sec). Part of the time course of liver sampling is seen in Fig. 5.

The time of cutting, defined as the time from the knife edge hitting the rat until it is cut through, amounts to $0.02-0.03 \mathrm{sec}$ depending partly on the pressure put on piston I (cf. Figs. 2 and $4 \mathrm{C}$ ) and partly on the part of the rat that has to be cut through (brain or liver sampling). The total sampling procedure, defined as the time from the knife edge hitting the rat until the clamping movement of the cooling tongs is terminated, i.e., until the beginning of the freezing, amounts to 0.09-0.11 sec.

\section{General}

Compared with the sampling techniques hitherto published, the present technique has the advantage of being about 30 times as rapid for liver sampling (3) and to allow regional studies on brain (7). However, the technique necessarily involves a cutting through of the tissue to be sampled, a mechanical stress which might produce biochemical artefacts. Especially concerning brain tissue, being an excitable tissue, this problem needs evaluation. ${ }^{3}$

3 The problem is being investigated at present in collaboration with Prof. B. K. Siesjö concerning an effect on metabolite concentrations and in collaboration with Prof. B. Chance concerning the effect on the redox state. 
The present method has only been worked out for liver and brain tissue sampling. However, the transverse section, the biopsy slice to be freezeclamped, can be made at any part (longitudinally) of the animal. Therefore, in principle, rapid sampling of any tissue from the rat is possible using the apparatus described. The limitation lies in the possibilities of isolation of the relevant tissue from the freezeclamped biopsy. Preliminarily, sections of the thorax have been tried, and it seems possible to harvest both heart and lung tissue from such freezeclamped sections of the thorax.

\section{ACKNOWLEDGMENTS}

The author thanks E. Petersen and A. Bilberg for the fabrication of the sampling apparatus. Thanks are also due to Prof. F. Lundquist for his interest in and encouragement of the work.

\section{REFERENCES}

1. Brosnan, J. T., Krebs, H. A., and Williamson, D. H. (1970) Biochem. J. 117, 91-96.

2. Hems, D. A., and Brosnan, J. T. (1970) Biochem. J. 120, 105-111.

3. Faupel, R. P., Seitz, H. J., Tarnowski, W., Thiemann, V., and Weiss, C. H. (1972) Arch. Biochem. Biophys. 148, 509-522.

4. Nilsson, L., and Siesjo, B. K. (1970) Acta Physiol. Scand. 80, 235-248.

5. Sietz, H. J., Faupel, R. P., Kampf, S. C., and Tarnowski, W. (1973) Arch. Biochem. Biophys. 158, 12-18.

6. Wollenberger, A., Ristau, O., and Schoffa, G. (1960) Pfluegers Arch. 270, 399-412.

7. Veech, R. L., Harris, R. L., Veloso, D., and Veech, E. H. (1973) J. Neurochem. 20, 183-188.

8. Quistorff, B. (1972) Commun. Meetings FEBS 8, abstract 828 (1972).

9. Bolwig, T, G., and Quistorff, B. J. Neurochem. 21, 1345-48 (1973).

10. Nordberg, K., Quistorff, B., and Siesjö, B. K. Acta Physiol. Scand., submitted for publication.

11. Ji, S., Chance, B., Welch, F., and Quistorff, B. (1975) Biophysical Society, Philadelphia. Abstract FAM-K5.

12. Quistorff, B., and Chance, B. (1975) Commun. Meetings FEBS lo, Paris July 1975. Abstract submitted.

13. Folbergrova, J., Nordberg, K., Quistorff, B., and Siesjo, B. K. (1975) J. Neurochem, in press. 